\title{
Establishing core outcome sets for gastrointestinal recovery in studies of postoperative ileus and small bowel obstruction: protocol for a nested methodological study
}

SJ Chapman ${ }^{1 *}, \mathrm{MJ}$ Lee $^{2,3 *}$, S Blackwell ${ }^{4}, \mathrm{R}$ Arnott ${ }^{5}$, RPG ten Broek ${ }^{6}, \mathrm{CP}$ Delaney ${ }^{7}$, NN DudiVenkata ${ }^{8}$, D Hind ${ }^{9}$, DG Jayne ${ }^{1}, K_{\text {Mellor }}^{9}$, A Mishra $^{10}$, G O’Grady ${ }^{11}$, T Sammour ${ }^{12}$ G Thorpe ${ }^{13}$, $\mathrm{Cl}$ Wells $^{14}$, AM Wolthuis ${ }^{15}$, NS Fearnhead ${ }^{16}$ on behalf of the Tripartite Gastrointestinal Recovery Group

${ }^{*}$ Denotes joint first authors

\section{Affiliations:}

1. Leeds Institute of Medical Research, University of Leeds, Leeds, UK

2. Academic Directorate of General Surgery, Sheffield Teaching Hospitals NHS Foundation Trust, Sheffield, UK

3. Department of Oncology \& Metabolism, Medical School, University of Sheffield, Sheffield, UK

4. Patient Representative, Liverpool, UK

5. Patient Representative, Green Templeton College, Oxford, UK

6. Department of Surgery, Radboud University Medical Center, Nijmegen, NL

7. Department of Colorectal Surgery, Digestive Disease Institute, Cleveland Clinic, Cleveland, Ohio, US,

8. Discipline of Surgery, Faculty of Health \& Medical Science, School of Medicine, University of Adelaide, Adelaide, AU

9. Clinical Trials Research Unit, University of Sheffield, Sheffield, UK

10. Department of Surgery, Maulana Azad Medial College, New Delhi, India 
11. Department of Surgery, Faculty of Medicine and Health Sciences, University of Auckland, Auckland, NZ

12. Colorectal Unit, Department of Surgery, Royal Adelaide Hospital, Adelaide, AU

13. Faculty of Medicine and Health Sciences, University of East Anglia Norwich, UK

14. Department of Surgery, Faculty of Medical \& Health Sciences, University of Auckland, Auckland, NZ

15. Department of Abdominal Surgery, University Hospital Leuven, Leuven, BE

16. Cambridge University Hospitals NHS Foundation Trust, Cambridge, UK

\section{Correspondence should be addressed to:}

Stephen J Chapman MRCS (Eng), NIHR Doctoral Research Fellow, Leeds Institute of Medical Research at St. James's, University of Leeds, United Kingdom, LS9 7TF.

Email: stephen.chapman@doctors.org.uk; Twitter: @SJ_Chapman

Running title: Developing core outcome sets for gastrointestinal recovery

Article Type: Study protocol

Manuscript word count: 2,511

Competing interests: None declared

Contributions: SJC, MJL, and NSF conceptualised the study idea and all authors provided input into its design and local set up. All authors contributed to draft versions of the manuscript and approved the final version for submission. NSF is the study guarantor.

Acknowledgements: The authors are grateful to all attendees of the Gastrointestinal Recovery Research Day (28 ${ }^{\text {th }}$ April 2017; Royal College of Surgeons of England, London), supported by the Association of Coloproctology of Great Britain \& Ireland Delphi research 
programme. The authors also thank member societies of the Tripartite 2020 Vision Initiative for their ongoing support in facilitating and promoting this study.

\section{Abstract}

Introduction: Gastrointestinal recovery describes the restoration of normal bowel function in patients with bowel disease. This may be prolonged in two common clinical settings: postoperative ileus and small bowel obstruction. Improving gastrointestinal recovery is a research priority but researchers are limited by variation in outcome reporting across clinical studies. This protocol describes the development of core outcome sets for gastrointestinal recovery in the contexts of postoperative ileus and small bowel obstruction.

Methods: An international Steering Group consisting of patient and clinician representatives was established. As overlap between clinical contexts is anticipated, both outcome sets will be co-developed and may be combined to form a common output with disease-specific domains. The development process will comprise three phases, including definition of outcomes relevant to postoperative ileus and small bowel obstruction from systematic literature reviews and nominal-group stakeholder discussions; online-facilitated Delphi surveys via international networks; and a consensus meeting to ratify the final output. A nested study will explore if the development of overlapping outcome sets can be rationalised.

Dissemination and implementation: The final output will be registered with the COMET initiative. A multi-faceted, quality improvement campaign for the reporting of gastrointestinal recovery in clinical studies will be launched, targeting international professional and patient 
groups, charitable organisations, and editorial committees. Success will be explored via an updated systematic review of outcomes five years after registration of the core outcome set.

\section{Introduction}

Gastrointestinal recovery describes the restoration of normal bowel function in patients receiving treatment for bowel disease. This may be prolonged in two settings. The first is postoperative ileus, characterised by reduced or uncoordinated intestinal transit. This occurs in $10-20 \%$ of patients undergoing major gastrointestinal surgery, depending on the criteria used for assessment (1). The second is small bowel obstruction, characterised by intestinal blockage. This is responsible for half of all emergency laparotomies in the United Kingdom and over 300,000 hospital admissions per year in North America (2, 3). Whilst both represent different aetiologies, the clinical manifestations of gastrointestinal recovery are similar.

Delayed gastrointestinal recovery is distressing for patients. It leads to painful abdominal distension, vomiting, delayed elimination, and prolonged hospital stay. It may also implicate the need for nasogastric decompression. The Association of Coloproctology in Great Britain \& Ireland recently identified gastrointestinal recovery as a key research priority during a patientclinician consensus process (4). Researchers are limited, however, by methodological challenges related to the definition of normal gastrointestinal function and the selection of clinical outcomes (5). Recently, two systematic reviews of previous literature identified 73 and 50 outcomes used to measure gastrointestinal recovery in settings of ileus and small bowel 
obstruction, respectively $(6,7)$. This wide variation in reporting limits comparability between studies, and is problematic for evaluating new treatments and translating these into practice.

The COMET (Core Outcome Measures in Effectiveness Trials) Initiative aims to develop 'core outcome sets' which represent the minimum information to be reported in all studies of a clinical condition (8). Currently, no such core outcome set exists for gastrointestinal recovery in either context of ileus or small bowel obstruction This would increase the value of future research through consistent and systematic reporting of results. As there is considerable overlap in the features of gastrointestinal recovery between both conditions, co-development of two outcome sets with the scope to combine or segment condition-specific domains represents an efficient approach. A protocol, analysis plan, and impact strategy are herein described.

\section{Methods}

This study protocol is reported with consideration to the Core Outcome Set-STAndards for Development (COS-STAD) recommendations and the Core Outcome Measures in Effectiveness Trials (COMET) handbook $(8,9)$. The final core outcome set will be reported according to the COS-STAndards for Reporting Statement (10). Research ethics approval will be confirmed in advance of all fieldwork and individual participant consent will be sought.

\section{Scope}

We aim to establish global consensus amongst patients, carers, academics, and clinicians on a minimal set of outcomes for gastrointestinal recovery in contexts of ileus after gastrointestinal surgery, and small bowel obstruction not requiring surgery. Both conditions represent different clinical contexts, but both share common challenges related to gastrointestinal recovery. We consider gastrointestinal surgery to represent any abdominal procedure performed on the intestinal tract, from the oesophagus (below the diaphragm) through to the anus. Other abdominal procedures (such as vascular, urological, and gynaecological) are excluded since unique speciality- and disease-specific outcomes may apply. 
An international, patient-clinician steering group has been established with representation from four geographical areas (Asia, Australasia, Europe, and America). Expert representatives have insights into the challenges of study design and outcome selection. Patient representatives have insights into the clinical and social implications of gastrointestinal recovery and will ensure that the patient perspective remains prominent throughout. All members of the steering group will be involved in the planning, execution, and dissemination of the final core outcome set.

\section{Stakeholders}

Patients and healthcare professionals will contribute equally to the development of the final core outcome set. To maximise generalisability and capture diverse viewpoints, contributions will be invited across a wide international setting, including (but not limited to) countries in Asia, Australasia, Europe, and North America. Patient and carer contributions will be invited from individuals with personal insights into ileus or small bowel obstruction. Professional contributions will be invited from individuals with expert insights into gastrointestinal recovery. This will include clinicians (gastrointestinal surgeons, anaesthesiologists, intensivists, and gastroenterologists), nurses (including nurse specialists such as stoma and enhanced recovery nurses), and allied healthcare professionals (dieticians and physiotherapists).

\section{Study Design}

The development of two outcome sets for gastrointestinal recovery (ileus and small bowel obstruction) will begin in parallel. Due to similarities between both clinical contexts, common domains may emerge and may justify the development of a modular outcome set. Decisions on the final structure will be informed through iterative review of emerging data. The development process will comprise three phases (Figure 1). In the first phase, outcomes identified from systematic reviews will be longlisted, followed by further additions during a series of nominal-group discussions. The second phase will consist of a three-round, online Delphi survey disseminated via international networks. In the third phase, a face-to-face stakeholder consensus meeting will be convened for voting on the final eligibility of short-listed 
outcomes. The study will be followed by a quality improvement campaign to disseminate and implement the final outcome set across stakeholder communities.

\section{Longlisting of Outcomes}

A longlist of outcomes reported in previous studies of ileus and small bowel obstruction will be compiled from two recent systematic reviews $(6,7)$. Further longlisting will take place via patient-clinician discussions (using nominal group technique) during a series of international study meetings. These will be small gatherings ( $<10$ individuals) convened jointly by video conference and local members of the Steering Group. A single meeting will take place for each geographical area (Asia, Australasia, Europe, and North America) to maximise the study's applicability. Additional outcomes generated during small group discussions will be added to produce two final longlists of outcomes for ileus and small bowel obstruction, respectively.

\section{Delphi Survey}

Individuals from all stakeholder groups will be invited to participate in a three-round, onlinefacilitated, international Delphi survey using the Google Forms platform. All surveys will be conducted in English. During the first round, surveys for ileus and small bowel obstruction will run in parallel and participants will be offered the opportunity to complete one or both; subsequent invitations will be issued according to this pre-specified preference. Invitations will be disseminated via professional societies, social media platforms, patient support groups, international collaborators, and charitable organisations. Consent to participate will be confirmed prior to completion of the first survey. Participants may offer novel outcomes via an open-ended question during the first Delphi round for incorporation into subsequent rounds of voting. Feedback on the process will also be sought. Participants who complete all Delphi rounds will be offered co-contributorship of the final output.

During each round, longlisted outcomes will appear in a random order. They will be linked to a plain English summary which will be co-authored and piloted by patient members of the Steering Group. In each round, participants will score outcomes using a Likert scale of 1-9, as 
recommended by the Grading of Recommendations Assessment, Development and Evaluation working group and COMET initiative $(8,11)$. Scores of $1-3$ will indicate an outcome of 'little importance', 4-6 will indicate 'some importance', and 7-9 will indicate 'great importance'.

During Round 1, scores will be analysed within each stakeholder group (patients, clinicians, nurses, and allied healthcare professionals). Consensus for short-listing of outcomes will be defined according to the following criteria.

- Greater than $70 \%$ of participants in at least one stakeholder group scoring the outcome as $7-9$ and less than $15 \%$ in at least one group scoring the outcome as $1-3$.

Responses will be collected across a period of 8 weeks. The Steering Committee will meet to discuss the inclusion of additional outcomes and rewording of survey components if misinterpretation is suspected from feedback. The results of both surveys (ileus and small bowel obstruction) will also be discussed; sufficient similarities in scoring may justify the development of a common outcome set with modules relating to specific clinical domains.

During Rounds 2 and 3, participants' previous responses, as well as a summary of stakeholderspecific scores from previous rounds will be presented. Only participants who contributed to the first round of surveys will be invited to participate in subsequent rounds. Participants will be asked to re-score all outcomes which have not yet met the criteria for short-listing. The same criteria for consensus will be applied across all rounds. All outcomes that reach consensus after three rounds will enter the short-list and will be considered at the consensus meeting. They will be considered together with items where split-voting, misinterpretation of language, or marked heterogeneity in panel prioritisation is noted by the Steering Group.

\section{Consensus Meeting}

Through a process of maximum variation purposive sampling, 10 representatives from each stakeholder group will be invited to a consensus event and asked to vote on the inclusion and 
exclusion of short-listed outcomes. This will be held at an international academic conference where attendance of all relevant stakeholders is anticipated. Representatives from journal editorial committees, industry, and surgical interest groups will also be invited as observers to provide comments on the final outcome set and its future implementation. Three rounds of voting with interactive discussion will be facilitated. Outcomes that exceed a pre-determined threshold of agreement $(70 \%)$ will enter the final outcome set.

\section{Final Core Outcome Set development}

It is envisaged that the final outcome set will consist of no more than 10 potential outcomes. Whilst there is no recommended maximum, an outcome set consisting of a rational number of outcomes is desirable to facilitate implementation. Each of the accepted outcomes will be categorised according to areas of the OMERACT filter (life impact; resource use; pathophysiological manifestations; and death) (12). Based on review of all data, the Steering Committee will decide on recommendations for the final structure; this may comprise two discrete core outcome sets for ileus and small bowel obstruction, or a common modular outcome set with domains relevant to both clinical contexts.

\section{Attrition and Sample Size}

It is anticipated that some participants will drop out from each round of the Delphi survey. Each participant will be allocated a unique identification number to allow tracking and identification of responses. The Steering Group will monitor trends in attrition (such as stakeholder group and geographical region) between rounds and consider strategies to maximise the response rates. There are no formal recommendations to guide sample size calculation for a Delphi survey, but a target of 100 international participants per stakeholder group will be sought. Recruitment to the first Delphi round will begin 8 weeks prior to the surveys opening for responses and no further participants will be included once the first round closes. 
The final outcome set will be registered with the COMET initiative. A quality improvement campaign for the reporting of gastrointestinal recovery in academic literature will be launched. This will involve presentations to academic audiences, lobbying of journal editorial teams, and promotion to professional organisations. Open-access plain English summaries will be disseminated to relevant patient and public groups. It is anticipated that uptake of the final output will be gradual. Success will be explored via an updated systematic review of outcomes five years after registration of the core outcome set. A $25 \%$ reduction in uniquely reported outcomes will represent a pragmatically meaningful improvement.

\section{Nested Methodological Study}

As this study considers two distinct clinical conditions albeit with some overlap in modes of presentation, a design modification to improve the efficiency of core outcome set development will be explored. This will involve Principal Component Analysis (PCA), a process which uses factor analysis to reduce the number of items in a list into themes measuring similar ideas or constructs (13). It achieves this by looking at the correlation of items across respondents and the behaviours of groups of items. In the present setting, PCA will be applied to both Delphi surveys (ileus and small bowel obstruction) after the conclusion of Round 1. Where components with similar constituent items are seen across both surveys, they will be merged for Round 2 and scored in combination. By measuring internal consistency of merged components, it may be possible to identify items with a high probability of exclusion. In the future, this may help participants to reduce the number of items within each domain more rapidly to produce a more consistent development process. A full description of these methods is outlined in Suppl. File $1(14,15)$. 


\section{Discussion}

Delayed gastrointestinal recovery in the context of ileus after intestinal surgery and small bowel obstruction is distressing for patients and costly for healthcare systems. Although the development of a core outcome set for gastrointestinal recovery will not lead to immediate changes to clinical care, it should lead to improved reporting of outcomes in the published literature. This will facilitate more valid comparisons of new treatments, helping to expedite improvements in the care offered to patients with these conditions. Importantly, the role of a core outcome set is to determine which outcomes are most relevant, as guided by a panel of key stakeholders. It should not command which outcome is most appropriate for calculating sample size, and neither can it guide how individual outcomes should be measured. Further research to refine these questions in the context of gastrointestinal recovery will be necessary.

The efficient development of a core outcome set for two clinical contexts is a strength of this study. Although ileus after gastrointestinal surgery and small bowel obstruction represent different clinical contexts, the challenges of gastrointestinal recovery between both are similar. An efficient and practical design may help with implementation of the final outcome set and is beneficial for avoiding research waste and duplication. Another strength of this study is the 
support from international and diverse stakeholders. Contributions from patients and carers ensures that the eventual outcome set remains patient-centred; support from multiple allied disciplines ensures that the outcome set is relevant to multidisciplinary practice; and involvement from collaborators from diverse geographical regions (including low-middle developed countries) ensures that it is widely generalisable.

Challenges in the study design are also recognised. Firstly, response rates for individual stakeholder groups in the Delphi surveys is a key challenge when facilitating the study online. It is possible that a disproportionate number of clinicians may respond to the survey, but this will be mitigated by working closely with patient support groups, charitable organisations, and patient members of the Steering Group. It is hoped that a balanced number of patient and carer contributions will be gathered to ensure that the process is patient-focussed throughout. Likewise, the Steering Group will ensure that the views of the patient stakeholder group are always accorded priority. Secondly, the scope of the study is limited to gastrointestinal recovery within the context of gastrointestinal disease, which does not account for other types of surgery where ileus is also problematic. This includes vascular, gynaecological, orthopaedic, cardiac, and urological procedures. This is necessary since is it possible that unique, disease-specific outcomes may apply to these patient groups but would not be captured from the current cohort of stakeholders. Finally, achieving wide implementation of a core outcome set is challenging and time consuming. This is a gradual process requiring extensive support from academic leaders and influencing bodies. A prospective impact and dissemination plan is presented and will ensure that issues of implementation are considered throughout. Measurable criteria for determining success of implementation will help to track progress of this plan and identify where further efforts may be needed in the future. 


\section{References}

1. Scarborough JE. Schumacher J. Kent KC. Heise CP. Greenberg CC. Associations of specific postoperative complications with outcomes after elective colon resection: a procedure-targeted approach toward surgical quality improvement. JAMA Surg 2017;152:e164681.

2. Ray NF, Denton WG, Thamer M, Henderson SC, Perry S. Abdominal adhesiolysis: inpatient care and expenditures in the United States in 1994. J Am Coll Surg 1998; 186: 1-9.

3. Stewart B, Khanduri P, McCord C, Ohene-Yeboah M, Uranues S, Vega Rivera F et al. Global disease burden of conditions requiring emergency surgery. Br J Surg 2014; 101: e9-e22.

4. Tiernan J, Cook A, Geh I. George B. Magill L. Northover J. et al. Use of a modified delphi approach to develop research priorities for the association of coloproctology of Great Britain and Ireland. Colorectal Dis 2014;16:965-975.

5. Chapman SJ. Wells Cl. Challenges in ileus research. Colorectal Dis 2018;20:639.

6. Chapman SJ. Thorpe G. Vallance AE. Harji DP. Lee MJ. Fearnhead NS. Association of Coloproctology of Great Britain and Ireland Gastrointestinal Recovery Group. Systematic review of definitions and outcome measures for return of bowel function after gastrointestinal surgery. BJS Open 2018;3:1-10. 
7. Mellor K. Hind D. Lee MJ. A systematic review of outcomes reported in small bowel obstruction research. J Surg Res 2018; 229:41-50

8. Williamson PR. Altman DG. Bagley $\mathrm{H}$. et al.. The COMET handbook: version 1.0. Trials $2017 ; 18: 280$.

9. Kirkham JJ. Davis K. Altman DG. et al. Core Outcome Set- STAndards for Development: The COS-STAD recommendations. PLoS Med 2017;14:e1002447.

10. Kirkham JJ. Gorst S. Altman DG. et al. Core outcome set- STAndards for reporting: the COSSTAR Statement. PLoS Med 2016;13:e1002148.

11. Guyatt GH. Oxman AD. Kunz R. et al. GRADE guidelines: 2. Framing the question and deciding on important outcomes. J Clin Epidemiol 2011;64:395-400.

12. Boers M. Kirwan JR. Gossec L. et al. How to choose core outcome measurement sets for clinical trials: OMERACT 11 approves filter 2.0. J Rheumatol 2014;41:1025-30

13. Lever J. Krzywinski M. Altman N. Points of Significance: Principal component analysis. Nature Methods 2017;14:641-42.

14. SAS Statistics. Principal Component Analysis [Resource Available from: http://support.sas.com/publishing/pubcat/chaps/55129.pdf. Accessed: $6^{\text {th }}$ June 2019.

15. Comrey A, Lee H. A first course in factor analysis. 2nd Ed ed. London: Routledge 1992. 


\section{Figure 1: Study schema:}

Phase 1: Finalisation of long list; Phase 2: three-round Delphi survey; Phase 3: Consensus meeting to finalise the outcome set. The study will be followed by a dedicated quality improvement campaign with a prospective plan for dissemination and implementation.

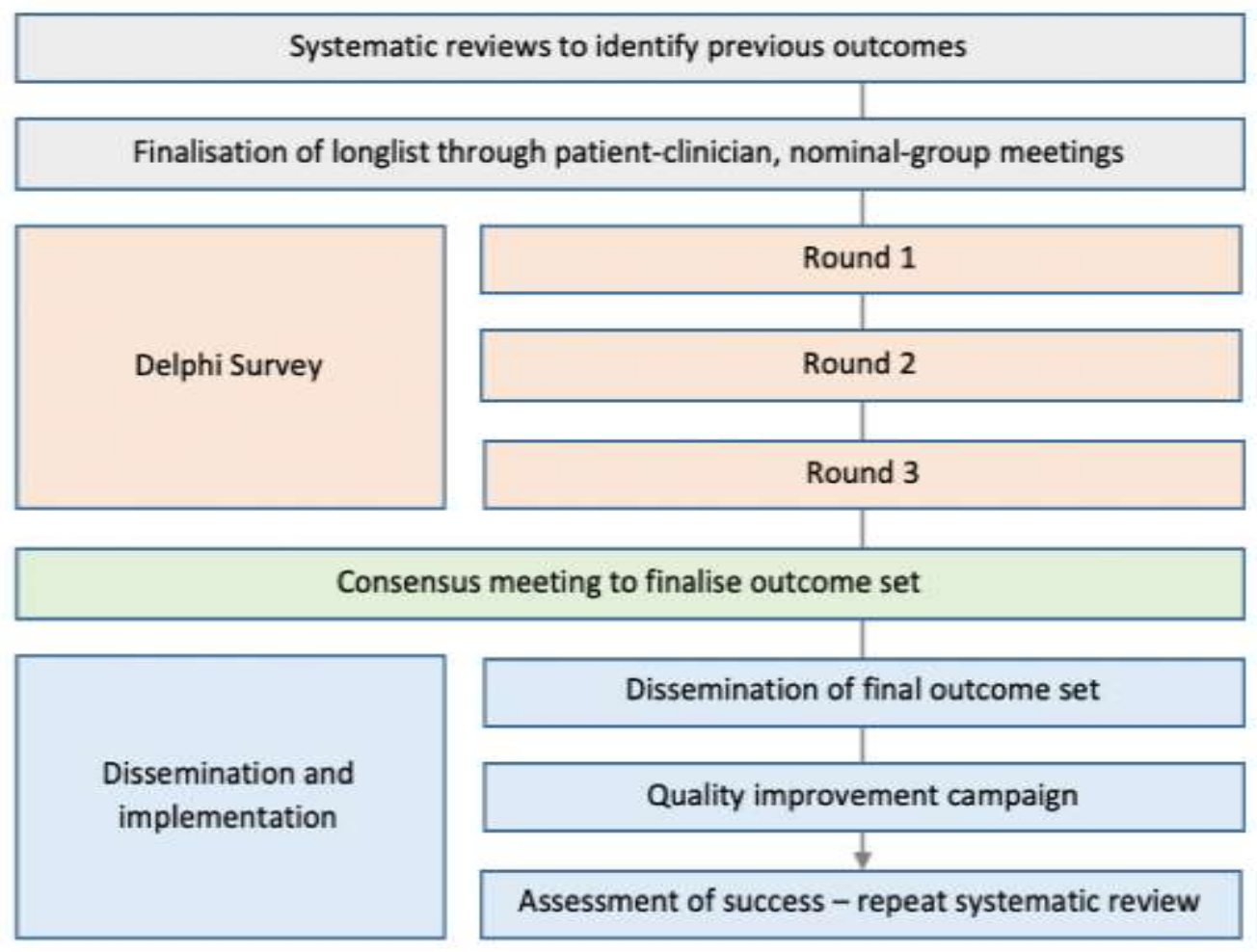

